\title{
Predictive Value of Platelet Indices in Development of Preeclampsia
}

Shaifali Dadhich, Sudesh Agrawal, Monica Soni, Rekha Choudhary, Rashmi Jain, Saroj Sharma, Sharad Lata Saini

\begin{abstract}
Aim: To evaluate the association between changes in platelet indices (platelet count, mean platelet volume, platelet distribution width) and development of preeclampsia.
\end{abstract}

Materials and methods: Two hundred pregnant women at 20 to 24 weeks of gestation with singleton pregnancy and normal blood pressure were enrolled after taking well-informed consent. At monthly intervals CBC (complete blood count) was done from 20 to 24 weeks till 40 weeks and 7 days after delivery. Data regarding changes in platelet indices with increasing gestation was collected and analyzed.

Results: Platelet count decreased significantly in patients with preeclampsia compared to normal pregnant patients (19.4\% vs $7.4 \%)$. Mean platelet volume increased significantly in preeclampsia patients (44.5\% vs $9.22 \%$ ). Increase in PDW was observed significantly in patients with preeclampsia $(47.19 \%$ vs $29.4 \%$ ).

Conclusion: Patients with preeclampsia are more likely to have significant decrease in platelet count, increase in PDW and MPV. These changes can be observed at an earlier gestational age than significant rise in BP can be observed and are directly proportional to progressive rise in hypertension. Thus, estimation of platelet indices can be considered as an early, simple and cost-effective procedure in the assessment of severity of preeclampsia.

Keywords: Pregnancy, Preeclampsia, Platelet indices.

How to cite this article: Dadhich S, Agrawal S, Soni M, Choudhary R, Jain R, Sharma S, Saini SL. Predictive Value of Platelet Indices in Development of Preeclampsia. J South Asian Feder Obst Gynae 2012;4(1):17-21.

Source of support: Nil

Conflict of interest: None declared

\section{INTRODUCTION}

Preeclampsia is among the commonest medical disorder during pregnancy, it complicates about 5 to $10 \%$ of pregnancies and continues to be a major cause of maternal and perinatal morbidity and mortality. ${ }^{1}$ It is a multisystem disease of unknown etiology and there is a constant search for better prognostic factors to predict the progression and severity of disease. Alterations in coagulation, fibrinolysis and platelet and vascular endothelial function are believed to play an important role in the pathogenesis of preeclampsia. The fall in the platelet count is most frequent abnormality and is probably due to consumption during low-grade intravascular coagulation. ${ }^{2}$ Mean platelet volume and platelet distribution width are significantly higher in patients with preeclampsia compared to those with normal pregnancy. ${ }^{3}$
Thus, the aim of the study is to assess the changes occurring in platelet indices (platelet count, mean platelet volume and platelet distribution width) in patients with preeclampsia with duration of gestation and establish there role as prognostic markers in assessing the severity of preeclampsia and giving an early prediction of progressive hypertension and severity of the disease.

\section{MATERIALS AND METHODS}

This prospective study was conducted in the Department of Obstetrics and Gynecology, PBM Hospital, SP Medical College, Bikaner from December 2007 to December 2008, on patients attending antenatal clinic and/or admitted in maternity wards. A total of 250 pregnant women at 20 to 24 weeks of gestation with singleton pregnancy and normal blood pressure were enrolled after taking well-informed consent. Data was collected and analyzed from 200 patients as 50 patients were either fulfilling the exclusion criteria or those who did not come for follow-up. The demographic details, such as age, weight, parity, residence, socioeconomic status were noted. Blood pressure was taken by auscultatory method in sitting position after making patient comfortable. Patients were considered hypertensive if diastolic BP was greater than or equal to 90 $\mathrm{mm} \mathrm{Hg}$ on two occasions 4 hours apart or single reading of $>110 \mathrm{~mm} \mathrm{Hg}$. They were further divided into mild and severe preeclampsia. Cases with systolic BP between 140 and 160 $\mathrm{mm} \mathrm{Hg}$ and diastolic BP between 90 and $110 \mathrm{~mm} \mathrm{Hg}$ were considered as mild and those with systolic BP greater than $160 \mathrm{~mm} \mathrm{Hg}$ and diastolic greater than $110 \mathrm{~mm} \mathrm{Hg}$ were considered as severe preeclampsia. Blood samples were collected and sent for estimation of platelet indices by performing complete blood count (CBC) with fully automated quantitative hematology analyzer sysmex; K-1000. At each visit $\mathrm{BP}$ recorded and weight was taken. At monthly intervals $\mathrm{CBC}$ was done from 20 to 24 weeks till 40 weeks and 7 days after delivery. The person maintaining the observations was blinded from the changes in BP. Patients developing hypertension during the study time were dealt by another set of clinicians and were called more frequently according to the severity of hypertension and were investigated and treated accordingly. The observer maintains the records of monthly CBC was completely blinded off from this aspect. The cases delivered in the hospital and observations were also made regarding mode of delivery and condition of baby. After completion of study the study group had patients who remained normotensive throughout pregnancy and patients who developed hypertension and further classified into mild and severe.

Date regarding changes in platelet indices with increasing gestation was collected and analyzed by applying ANOVA test, 
Chi-square test, student t-test and inferences were made accordingly. $\mathrm{p}$-value $<0.001$ was considered as significant. The study has been approved by institutional ethical committee.

\section{RESULTS}

Total number of patients analyzed was 200, out of which 26 developed preeclampsia thus giving an incidence of $13 \%$. Out of these 26 preeclampsia patients 23 were mild and three were with severe preeclampsia. The mean age range of the pregnant women in the present study was $23.45 \pm 3.23$ years.

Table 1 shows distribution of preeclampsia patients with known risk factors of preeclampsia, $53.84 \%$ of patients in the preeclampsia group were primigravida. Patients developing preeclampsia had greater association with history of hypertension in previous pregnancy $(19.23 \%)$ and bad obstetric history $(11.53 \%)$.

Platelet count decreased during the course of normal pregnancy with period of gestation but it was statistically non significant. While significant and rapid decline in platelet count was observed in patients with preeclampsia and the decline was directly proportional to the severity of hypertension. The significant decrease in platelet count was antedating significant increase in BP by 4 to 6 weeks. Table 2 shows comparison of changes in platelet count between normotensive pregnant patients and preeclampsia patients. Monthwise decrease in platelet count in normotensive pregnant patients group was nonsignificant while in preeclampsia group highly significant decrease in platelet count occurred between 36 and 40 weeks and when monthwise comparisons between two groups were made it was seen that mean platelet count was less in preeclampsia group at each month as compared to normotensive pregnant patients group, and this decrease was more significant as duration of pregnancy increased. Table 3 shows comparison of changes in platelet count with duration of gestation between mild and severe preeclampsia patients. Platelet count was lower in mild preeclampsia patients and lowest in severe preeclampsia patients. The decrease in platelet count with progression of gestation was also more significant as severity of preeclampsia increased from mild-to-severe and highly significant decrease in platelet count was observed earlier (32 to 36 weeks) in severe preeclampsia patients as compared to mild preeclampsia patients (36 to 40 weeks).

Mean platelet volume increased in normal pregnancy but increase was nonsignificant. While significant and consistent increase was observed in preeclampsia patients occurring 4 to 6 weeks before a significant rise in BP could be observed. The maximum increase in MPV was observed in severe preeclampsia patients showing its direct relationship with

\begin{tabular}{|c|c|c|}
\hline Risk factors & No. of patients & Percentage \\
\hline Primigravida & 14 & 53.84 \\
\hline $\begin{array}{l}\text { History of hypertension in } \\
\text { previous pregnancy }\end{array}$ & 5 & 19.23 \\
\hline Bad obstetric history & 3 & 11.53 \\
\hline
\end{tabular}

increase in BP. Table 2 shows comparison of changes in mean platelet volume between normotensive pregnant patients and preeclampsia patients. Monthwise increase in MPV in normotensive pregnant women group was observed which was nonsignificant while in preeclampsia group monthwise increase in MPV was observed which was statically highly significant at 36 to 40 weeks of gestation and when monthwise comparisons were made between two groups mean value of mean platelet volume was more in preeclampsia group for each month. Table 3 shows monthwise comparison of mean platelet volume between mild and severe preeclampsia patients. Mean platelet volume was greater in mild preeclampsia patients and greatest in severe preeclampsia patients. The increase in mean platelet volume with progression of gestation was also more significant as severity of preeclampsia increased from mildto-severe and significant increase in mean platelet volume occurred earlier (32 to 36 weeks) in severe preeclampsia patients as compared to mild preeclampsia patients (36 to 40 weeks).

Platelet distribution width increase in normal pregnancy but nonsignificantly till 32 weeks while in preeclampsia patients the PDW values were higher and significant increase could be observed with increasing severity of hypertension and significant changes in PDW were seen before a significant rise in BP could be appreciated. Table 2 shows comparison of changes in platelet distribution width between normotensive pregnant patients and preeclampsia patients. In normotensive pregnant women group there was monthwise increase in PDW which was significant at 32 to 36 and 36 to 40 weeks and in preeclampsia group also there was monthwise increase in PDW which was highly statically significant at 32 to 36 weeks and 36 to 40 weeks and significance was more in preeclampsia group as compared to normotensive group. When monthwise comparison was done between two groups the mean value of PDW was higher in preeclampsia group than normotensive group at each period of gestation. Table 3 is demonstrating monthwise comparison of platelet distribution width between mild and severe preeclampsia patients. Platelet distribution width was greater in mild preeclampsia patients and greatest in severe preeclampsia patients at each period of gestation. The increase in platelet distribution width with progression of gestation was also more significant as severity of preeclampsia increases from mild-to-severe and significant increase in platelet distribution width occurred earlier (32 to 36 weeks) in all groups which continued up till 40 weeks and changes were observed before significant rise in BP was seen.

Table 4 shows correlation between blood pressure and three platelet parameters. The highest negative correlation index was found between platelet count and blood pressure. Positive correlation was established between BP, MPV and PDW.

\section{DISCUSSION}

Pregnancy is associated with complex and still incompletely understood changes involving blood coagulation. Information regarding behavior of platelets in normal pregnancy has shown varying trends. The study evaluated the serial changes occurring 


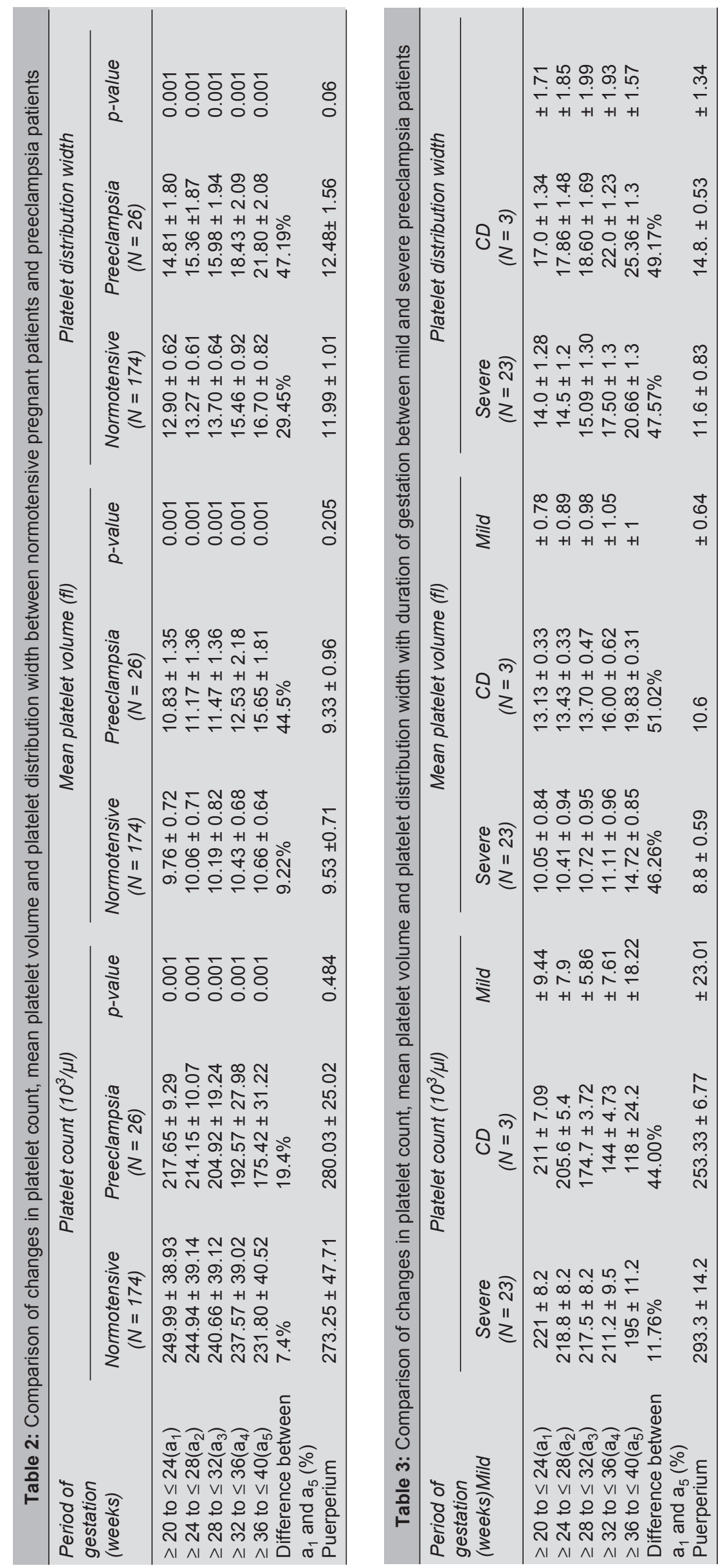


Table 4: Correlation index between blood pressure and platelet count, mean platelet volume and platelet distribution width in patients with preeclampsia

\begin{tabular}{|c|c|c|c|c|c|c|}
\hline \multirow{2}{*}{$\begin{array}{l}\text { Period of gestation } \\
\text { (weeks) }\end{array}$} & \multicolumn{2}{|c|}{ Platelet count $\left(10^{3} / \mu l\right)$} & \multicolumn{2}{|c|}{ Mean platelet volume (fI) } & \multicolumn{2}{|c|}{ Platelet distribution width } \\
\hline & Correlation index (r) & $p$-value & Correlation index (r) & $p$-value & Correlation index (r) & $p$-value \\
\hline$\geq 20$ to $\leq 24\left(a_{1}\right)$ & -0.496 & $<0.005$ & +0.807 & $<0.001$ & +0.619 & $<0.001$ \\
\hline$\geq 24$ to $\leq 28\left(a_{2}\right)$ & -0.638 & $<0.001$ & +0.767 & $<0.001$ & +0.636 & $<0.001$ \\
\hline$\geq 28$ to $\leq 32\left(a_{3}\right)$ & -0.876 & $<0.001$ & +0.760 & $<0.001$ & +0.632 & $<0.001$ \\
\hline$\geq 32$ to $\leq 36\left(a_{4}\right)$ & -0.892 & $<0.001$ & +0.880 & $<0.001$ & +0.655 & $<0.001$ \\
\hline$\geq 36$ to $\leq 40\left(a_{5}\right)$ & -0.868 & $<0.001$ & +0.851 & $<0.001$ & +0.819 & $<0.001$ \\
\hline
\end{tabular}

in platelet count, mean platelet volume and platelet distribution width with gestational age in normotensive pregnant patients and pregnant patients with preeclampsia. Significant differences were obtained. The present study also established a correlation index between BP and platelet count, mean platelet volume and platelet distribution width at each month of gestation respectively.

In the present study, $13 \%$ of patients developed preeclampsia. A total of $88.46 \%$ of preeclampsia patients belonged to mild category while $11.54 \%$ were with severe preeclampsia. At the end of the study when 26 preeclampsia patients were evaluated $53.84 \%$ of them were primigravida. Similar higher incidence of preeclampsia in primigravida patients has been reported by other authors. ${ }^{4}$

This study demonstrated an association between preeclampsia patients and history of hypertension in previous pregnancy of $19.23 \%$. In a study conducted by Sonia Hernandez et al, also showed increased risk of preeclampsia in subsequent pregnancies after having it in previous pregnancy. ${ }^{5}$

The present study also showed an association between patients with bad obstetric history and preeclampsia (11.53\%); as compared to normotensive pregnant patients and $\mathrm{BOH}$ $(4.5 \%)$. In their study Xiong Xu et al also found an increased incidence of preeclampsia in patients with previous two abortions and that a previous term pregnancy is protective factor against development of preeclampsia in future pregnancy. ${ }^{4}$

This present study demonstrated decrease in platelet count in normotensive pregnant patients with gestational age when counts at monthly intervals from 20 weeks to 40 weeks were compared (7.4\%). But statistically significant decrease (19.4\%) in platelet count was observed in patient with preeclampsia and decrease was highly significant at 36 to 40 weeks. Similar findings were seen in studies conducted by Leduc Line et $\mathrm{al}^{6}$ and Vrunda et al. ${ }^{7}$

It was evident that frequency and intensity of maternal thrombocytopenia depend upon the intensity of the disease and reduction in platelet count was an early feature of the disease and was directly proportional to severity of hypertension. Hence when monthwise estimations were done, fall in platelet count was statistically more significant as severity of preeclampsia increased $(11.76 \%$ in mild preeclampsia $v s 44 \%$ in severe preeclampsia). These significant changes in platelet count were observed even before significant rise in BP was seen. On reviewing literature, we found similar findings in the studies conducted by Vrunda et al. ${ }^{7}$ They reported platelet count 140 $\times 10^{9} / \mathrm{L}$ in severe PIH while it was $200 \times 10^{9} / \mathrm{L}$ in mild PIH and $220 \times 10^{9} / \mathrm{L}$ in normotensive patients. Yin SM et al also reported similar findings in their study on platelet function in $\mathrm{PIH}^{8}{ }^{8}$ In this study platelet count in PIH were $181 \pm 56 \times 10^{9} / \mathrm{L}$ as compared to $228 \pm 56 \times 10^{9} / \mathrm{L}$ in control group. Mohapatra et al also reported that degree of thrombocytopenia is directly proportional to severity of preeclampsia. ${ }^{9}$ In this study platelet counts were $182 \times 10^{9} / \mathrm{L}$ in severe PIH and $223 \times 10^{9} / \mathrm{L}$ in mild PIH and $238 \times 10^{9} / \mathrm{L}$ in normal subjects. Vamseedhar et al in their study observed similar findings. ${ }^{10}$ In this study platelet counts were $155 \times 10^{9} / \mathrm{L}$ in preeclampsia and $218 \times 10^{9} / \mathrm{L}$ in normal pregnant females.

In this study, MPV increased in normal pregnancy from 20 weeks onwards till 40 weeks but increase was not significant $(9.22 \%)$. The present study observed that there was a significant increase in MPV in preeclampsia patients $(44.5 \%)$ with duration of gestation as blood pressure increased. The increase was more significant as severity of preeclampsia increased (46.26\% in mild preeclampsia vs $51.02 \%$ in severe preeclampsia). Similar findings were reported by different authors. ${ }^{8,11-13}$ Yin SM et al observed a MPV of $11.2 \pm 2.0 \mathrm{fl}$ in PIH patients while it was $8.7 \pm 2.0 \mathrm{fl}$ in normotensive patients. ${ }^{8}$ Giles et al found MPV $9.9 \mathrm{fl}$ in preeclampsia patients and $8.7 \mathrm{fl}$ in normal pregnant subjects. ${ }^{14}$

In patients with mild preeclampsia significant increase was seen at 36 to 40 weeks while patients severe preeclampsia showed increase at 32 to 36 weeks which continued till 40 weeks. Significant increase in MPV can be detected in patients weeks prior to diagnosis of PIH. This has been shown by Ozgur et al who found significant increase in MPV which preceded the diagnosis of PIH by approximately 4.6 weeks (range 2.8 to 5.9 weeks). ${ }^{11}$ Similarly, Walker et al observed significantly increased MPV 1 week before BP became clinically significantly apparent. ${ }^{12}$

We found steady and significant increase in platelet distribution width which occurred at 32 to 36 weeks and continued till 40 weeks $(29.4 \%)$ in normotensive pregnant patients. PDW in patients with preeclampsia increase and significant increase $(47.19 \%)$ is seen at 32 to 36 weeks which continued till 40 weeks. Previously similar findings were reported by studies conducted by various authors. ${ }^{3,15,16}$ Giles et al in their study found PDW $16 \mathrm{fl}$ in PIH and 12 in normal pregnant females. ${ }^{14}$ Recently, Vamseedhar in his study on significance of platelet indices in PIH observed PDW $15.51 \mathrm{fl}$ in preeclampsia and $11.07 \mathrm{fl}$ in normal pregnancy. ${ }^{10}$

A direct relationship between platelet distribution width and severity of preeclampsia was observed. Significant increase was observed with increase in blood pressure $(47.57 \%$ in mild 
preeclampsia $v s 49.17 \%$ in severe preeclampsia). The rise in PDW was observed before significant rise in BP could be seen. Hence PDW serves as a reliable indicator of severity of preeclampsia.

There is a constant search for better prognostic factors to predict severity of disease. To achieve this correlation index was calculated for each parameter and relationship between $\mathrm{BP}$ and each of these three parameters, i.e. platelet count, MPV and PDW was established.

A negative correlationship was seen between BP and platelet count and as the BP increased significantly the r-value (correlation index) also increased proportionally and was highest at 36 to 40 weeks (r-value $-0.868=$ p-value $<0.001$ ) when rise in BP was also maximum. We observed a positive correlation index for MPV and PDW with BP (r-value 0.851 for MPV and r-value 0.819 for PDW). These correlation indices also increased proportionally with increase in BP and were highest at 36 to 40 weeks. Hence, these parameters can be used as useful sensitive and specific prognostic markers in predicting the severity of preeclampsia and detecting patients who are likely to develop progressive hypertension thus requiring an early intervention.

\section{CONCLUSION}

Patients with preeclampsia are more likely to have rapid and significant decrease in platelet count, increase in PDW and MPV in comparison to the normotensive counterparts. These significant changes can be observed at an earlier gestational age than significant rise in BP can be observed and changes are more significant in patients who are destined to develop progressive severe hypertension. Changes in these three parameters can be correlated with changes in BP and hence, prediction of patients likely to develop progressive hypertension can be done earlier, and interventions done accordingly. Thus estimation of platelet indices seems to be a reliable, rapid, easy and economical method for early detection of preeclampsia and assessment of its severity.

\section{REFERENCES}

1. Roberts JM,Cooper DW. Pathogenesis and genetics of preeclampsia. Lancet 2001;357(9249):53-56.

2. Halligan A, Bonnar J, Sheppard B, et al. Haemostatic, fibrinolytic and endothelial variables in normal pregnancies and preeclampsia. Br J Obstet Gynaecol 1994;101:488.

3. Rosevear SK, Ligginis GC. Platelet dimensions in pregnancy induced hypertension. New Zealand Med J 1986;99(802): 356- 57.

4. Xiong, Xu, William D, et al. History of abortion, preterm and term birth and risk of preeclampsia: A population-based study. Am Journal of Obstetric and Gynecology 2002;187(4):1013-18.

5. Diaz Sonia Hernandez, Toh Sengwee, et al. Risk of preeclampsia in first and subsequent pregnancies: A prospective cohort study. BMJ 2009;338:b2255.

6. Leduc Line, Wheeler, James M, et al. Coagulation profile in severe preeclampsia, Obstetrics and Gynecology 1992;79(1):14-18.

7. Joshi Kale Vrundra, Sapre Shaila. Lowered platelet count: A prognostic index in preeclampsia. J Obstet Gynaecol Ind 2004; 54(3):235-36.
8. Yin SM, Li Yq, Feng JH, et al. Study on the variation of platelet function in pregnancy induced hypertension and gestational diabetes mellitus. Hematology 2005;40(1):25-28.

9. Mohapatra S, Pradhan BB, Satpathy UK, Mohanty A, Pattnaik JR. Platelet estimation: Its prognostic value in pregnancy induced hypertension. Ind J Physiol Pharmacol 2007;51(2):160-64.

10. Vamseedhar Annam, Srinivasa K, Santhosh K Yatnatti, Suresh DR. Evaluation of platelet indices and platelet counts and their significance in preeclampsia and eclampsia. Int J Biol Med Res 2011;2(1):425-28.

11. Ozgur Dundar, Pinar Yoruk. Longitudinal study of platelet size changes in gestation and predictive power of elevated MPV in diagnosis of preeclampsia. Prenatal Diag 2008;28(11):1052-56.

12. Walker JJ, Cameron AD, Bjornsson S, Singer CRJ, Fraser C. Can platelet volume predict progressive hypertensive disease in pregnancy? Am J Obstet Gynaecol 1989;161;676-79.

13. Ahmed Y, Iddekinge BV, Paul C, Sullivan MHF, Elder MG. Retrospective analysis of platelet numbers and volumes in normal pregnancy and in preeclampsia. Br J Obstet Gynecol 1993;100: 216-20.

14. Giles C. The platelet count and mean platelet volume. Br J Haematol 1981;48:31-37.

15. Santos, dos Elvany Veronica, Jose Meirelles Filho. Measurement of platelet parameters in normal and preeclamptic pregnant women. Rev Bras Ginecol Obstet 2004;26(3):201-06.

16. Tygart SG, McRoyan DK, Spinnato JA, Kitay DZ. Longitudinal study of platelet indices during normal pregnancy. Am J Obstet Gynecol 1986;154:883-87.

\section{ABOUT THE AUTHORS}

\section{Shaifali Dadhich (Corresponding Author)}

Senior Resident, Department of Obstetrics and Gynecology SP Medical College, Bikaner-334003, Rajasthan, India, Phone: +919214018793, e-mail: shaifalidr23@gmail.com

\section{Sudesh Agrawal}

Associate Professor, Department of Obstetrics and Gynecology SP Medical College, Bikaner, Rajasthan, India

\section{Monica Soni}

Assistant Professor, Department of Obstetrics and Gynecology SP Medical College, Bikaner, Rajasthan, India

\section{Rekha Choudhary}

Senior Resident, Department of Obstetrics and Gynecology SP Medical College, Bikaner, Rajasthan, India

\section{Rashmi Jain}

Senior Resident, Department of Obstetrics and Gynecology SP Medical College, Bikaner, Rajasthan, India

\section{Saroj Sharma}

Medical Officer, Department of Obstetrics and Gynecology SP Medical College, Bikaner, Rajasthan, India

\section{Sharad Lata Saini}

Medical Officer, Department of Obstetrics and Gynecology SP Medical College, Bikaner, Rajasthan, India 